\title{
Activation of the LINC00242/miR-141/FOXC1 axis underpins the development of gastric cancer
}

\author{
Xiongdong Zhong ${ }^{*} \mathbb{D}$, Xianchang Yu, Xiaoyan Wen, Lei Chen and Ni Gu
}

\begin{abstract}
Background: Long non-coding RNAs (LncRNAs) are a class of newly identified transcripts recognized as critical governors of gene expression during human carcinogenesis, whereas their tumor-suppressive or tumor-promoting effects on gastric cancer (GC) are required for further investigation. In the study, we identify the expression pattern of a novel IncRNA LINC00242 in GC and its possible permissive role in the development of GC.

Methods: The study included 68 pairs of GC and adjacent normal gastric tissue samples. The viability, migration, and invasion of cultured human GC cells HGC27 were evaluated by CCK-8 and Transwell chamber assays. In vitro tube formation of human brain microvascular endothelial cells (HBMVECs) in HGC27 cell coculture was detected. The regulatory network of LINC00242/miR-141/FOXC1 was verified using dual luciferase reporter gene assay and RNA immunoprecipitation (RIP) assay. Subcutaneous xenografts of HGC27 cells were performed in nude mice.

Results: LINC00242 was highly expressed in GC tissues and cells and contributed to poor prognosis. LINC00242 knockdown inhibited HGC27 cell viability, migration and invasion, and tube formation of HBMVECs. LINC00242 interacted with miR-141 and positively regulated FOXC1, a target gene of miR-141. LINC00242 knockdown was partially lost in HGC27 cells upon miR-141 inhibition or FOXC1 overexpression. The tumor-promoting effect of LINC00242 on GC was demonstrated in nude mice.
\end{abstract}

Conclusion: Taken together, the present study demonstrates the oncogenic role of the LINC00242/miR-141/FOXC1 axis in GC, highlighting a theoretical basis for GC treatment.

Keywords: Gastric cancer, LINC00242, MicroRNA-141, FOXC1

\section{Background}

According to Global Cancer Statistics 2018, gastric cancer (GC) ranks the 5 th most frequently occurring cancer, accounting for $5.7 \%$ in both sexes on a global scale, and responsible for $8.2 \%$ of total cancer-related deaths [1]. It has been reported that there are several common therapeutic schedules for GC, such as surgery, radiotherapy, chemotherapy, and targeted therapy [2]. Although there

*Correspondence: ZhongxiongdongZXD@163.com

Department of General Surgery, Zhuhai People's Hospital (Zhuhai hospital affiliated with Jinan University), No.79 Kangning Road, Xiangzhou District,

Zhuhai 519000, Guangdong, China were great advancements in the treatment for $\mathrm{GC}$, the survival rate for advanced GC remains poor [3]. Accumulating evidence has revealed that GC is often accompanied by angiogenesis [4]. Angiogenesis is a complicated process to form new blood vessels from pre-existing vessels, and disruption of tumor angiogenesis could affect tumor growth and metastasis [5]. Thus, identification of the critical molecules involved in angiogenesis may provide breakthroughs for $\mathrm{GC}$ treatment.

LncRNAs exert their functions at the translational, transcriptional, and posttranscriptional level and are implicated in the occurrence and development of many human cancers, including GC [6]. Moreover, it's 
interesting to note that multiple lncRNA may be playing an important role in angiogenesis in GC [7], while roles of IncRNA LINC00242 in GC remains unclear. microRNAs (miRNAs), a group of small non-coding RNA molecules, serve as regulators of inhibition of mRNA translation or reduction of mRNA stability by binding to the $3^{\prime}$-untranslated region (3'-UTR) of target mRNA [8]. miR-141, belongs to the miR-200 family, is poorly expressed in GC and implicated in the GC development by regulating cell proliferation, invasion, and metastasis [9]. Transcriptome analysis demonstrates the presence of miRNA-IncRNA-mRNA interaction in the malignant transformation process of GC initiation [10]. For example, Zhou et al. have validated that IncRNA H19 competed with miR-141 exerts effects on GC [11]. A bioinformatics website predicts that forkhead box (Fox) $\mathrm{C} 1$ is a potential target gene of miR-141. FOXC1 is also proved to be participated in GC [12]. FOXC1, a member of the FOX family, is essential for cell proliferation, migration, invasion, as well as vascular formation and maturation $[13,14]$. However, their specific mechanism in angiogenesis of GC remains largely unknown. In the present study, the role of the newly discovered lncRNA LINC00242 in the cell invasion, metastasis, and angiogenesis of GC and its possible mechanisms is explored, which may help to provide a novel direction for GC treatment.

\section{Materials and methods}

\section{Ethics statement}

The Ethics Committee of Zhuhai People's Hospital has approved the study protocol. We receive informed written consents from all participants and do our best to minimize the number and suffering of animals.

\section{Tissue specimen collection}

Tumor tissues and their matched normal tissues adjacent to tumor were surgically resected from $68 \mathrm{GC}$ patients who were admitted into the Zhuhai People's Hospital from January 2013 to December 2015. None of the included patients received chemotherapy or radiotherapy. The 68 patients were seen for follow-up visits for 5-60 months and 9 patients were lost to follow-up.

\section{Cell harvest and transient transfection}

Human GC cell lines, SGC7901, BGC823, MKN45, HGC27, and a normal gastric epithelial cell line GES-1 (ATCC, USA) were harvested in DMEM medium supplemented with $10 \%$ fetal bovine serum (FBS) (Gibco, USA), $100 \mathrm{U} / \mathrm{mL}$ penicillin, and $100 \mathrm{mg} / \mathrm{mL}$ streptomycin $\left(5 \% \mathrm{CO}_{2}, 37{ }^{\circ} \mathrm{C}\right)$. Cultured HGC27 cells were treated with miR-141 mimic (\#4464066), miR-141 inhibitor (\#4464084), three anti-LINC00242 siRNA constructs (si-LINC00242-1, si-LINC00242-2, and
si-LINC00242-3), expression vectors containing the FOXC1 gene (oe-FOXC1), and their negative control (NC) [mimic-NC, inhibitor-NC, scramble siRNA (si-NC), and empty vector (oe-NC)] (all purchased from Thermo Fisher Scientific, USA) alone or in combination as required by using lipofectamin 2000 reagents (11668-019, Invitrogen, USA) for $48 \mathrm{~h}$ according to the manufacturer's instructions. The sense of siLINC00242: 5'-UAUCUCCAAGGCAUGGAGC-3'; the anti-sense of si-LINC00242: 5'-GCUGGAUGCCUU GGAGAUA- $3^{\prime}$. The sense of si-NC: $5^{\prime}$-UAUGGCUCG AACUACGAGC-3'; the anti-sense of si-NC: $5^{\prime}-\mathrm{GCU}$ CGUAGUUCGAGCCAUA- $3^{\prime}$.

\section{RNA isolation and quantification}

Total RNA was extracted from GC tissues or HGC27 cells by using the TRIZOL kit (Invitrogen, USA) following the manufacturer's instructions. Then, miRNA was reversely transcribed to cDNA with reference to the manual of TaqMan MicroRNA Assays Reverse Transcription prime whereas mRNA was reversely transcribed to cDNA according to instructions of EasyScript First-Strand cDNA Synthesis SuperMix (AE301-02, TransGen Biotech, Beijing, China). Reverse transcription quantitative polymerase chain reaction (RT-qPCR) was executed following the steps described in the instructions of $\mathrm{SYBR}^{\circledR}$ Premix Ex TaqTM II kit (TaKaRa, Dalian, Liaoning, China) by using ABI 7500 instrument (Applied Biosystems, Foster City, CA, USA). The expression of miR-141 was relative to U6 while that of the other genes was obtained with GAPDH as a loading control. The synthesis of primer sequences was committed to Shanghai Majorbio Co., Ltd., (Shanghai, China) (Table 1). Finally, the fold changes were calculated by means of relative quantification ( $2^{-\Delta \Delta \mathrm{Ct}}$ method).

\section{Table 1 Primer sequences used for RT-qPCR}

\begin{tabular}{|c|c|}
\hline Target & Primer sequences $\left(5^{\prime}-3^{\prime}\right)$ \\
\hline \multirow[t]{2}{*}{ LINC00242 } & F:TTCAGGCGCTGTCTGTTCTT \\
\hline & R:TGCGAATCGATGGGGATCAG \\
\hline \multirow[t]{2}{*}{ FOXC1 } & F: ACTCGGTGCGGGAGATGTT \\
\hline & R: CCTTGATGGGTTCCTTTAGC \\
\hline \multirow[t]{2}{*}{ miR-141 } & F: ACACTCCAGCTGGGCATCTTCCAG \\
\hline & R: CTCAACTGGTGTCGTGGAGTCGGC \\
\hline \multirow[t]{2}{*}{ U6 } & F: CTCGCTTCGGCAGCACA \\
\hline & R: AACGCTTCACGAATTTGCGT \\
\hline \multirow[t]{2}{*}{ GAPDH } & F:TGAAGGTCGGAGTCAACGG \\
\hline & R: CTGGAAGATGGTGATGGGATT \\
\hline
\end{tabular}

$F$ forward, $R$ reverse 


\section{Western blot analysis}

GC tissue homogenates or HGC27 cells were lysed by Radio Immunoprecipitation Assay lysis (P0013B, Beyotime Institute of Biotechnology Co., Ltd., Shanghai, China) supplemented with $1 \mathrm{mM}$ phenylmethylsulfonyl fluoride. The protein sample was separated by the method of the SDS-PAGE and transferred onto a polyvinylidene fluoride membrane. The membrane was probed with diluted primary antibodies (Abcam Inc., Cambridge, UK) [N-cadherin (1:1000, ab18203), Vimentin (1:1000, ab92547), MMP-2 (1:1000, ab37150), MMP-9 (1:1000, ab73734), VEGF (1:1000, ab53465), CD31 (1:1000, ab134168), GAPDH (1:1000, ab181602) overnight at $4{ }^{\circ} \mathrm{C}$. Immunoblots were visualized using goat anti-rabbit immunoglobulin G (IgG) (1:20000, ab6721, Abcam Inc.) and the enhanced chemiluminescence reagent. The gray values were assessed by means of Image J software (National Institutes of Health, Bethesda, Maryland).

\section{Cell viability assays}

Cells were seeded into 96-well plates $\left(1 \times 10^{5}\right.$ cells $\left./ \mathrm{ml}\right)$ and then allowed to react with $10 \mu \mathrm{l} \mathrm{CCK-8} \mathrm{(35000,} \mathrm{AAT}$ Bioquest, Mercury Drive, Sunnyvale, CA, USA) every other day at $37{ }^{\circ} \mathrm{C}$. Four hours later, the medium was replaced by $150 \mu \mathrm{L}$ Dimethyl Sulfoxide (DMSO, SigmaAldrich, USA) in each well to dissolve the formazan crystals. Absorbance was read at $450 \mathrm{~nm}$ using a Microplate reader (DNM-9602G; Aolu Biotech, Shanghai, China).

\section{Dual luciferase reporter gene assay}

Artificially synthesized FOXC1 3'untranslated region (UTR) fragments containing putative miR-141 binding sites were inserted into the pmirGLO (Promega, USA) (named FOXC1-WT). A complementary sequence mutation site of putative miR-141 binding sites was designed on FOXC1 3 'UTR and likewise inserted into the pmirGLO (named FOXC1-MUT). HEK293T cells (Shanghai Beinuo Biotech Ltd., Shanghai, China) were seeded into 6-well plates in triplicate and co-transfected with well-designed pmirGLO-based reporter plasmids and miR-141 mimic using the dual-luciferase reporter assay system according to the manufacturer's instructions (D0010, Beijing Solarbio Science \& Technology Co., Ltd, China). The luminescence of the firefly luciferase was normalized to the luminescence of the renilla luciferase.

\section{Transwell invasion and migration assays}

Transwell invasion assays were performed to detect cell invasion. The $200 \mu \mathrm{L}$ serum-free medium was added with $200 \mu \mathrm{L}$ Matrigel. Cells were prepared into cell suspension using medium containing 20\% FBS. Each well in the apical chamber-coated with Matrigel was added with $200 \mu \mathrm{L}$ cell suspension, and the basolateral chamber was added with $800 \mu \mathrm{L}$ conditioned medium containing $20 \% \mathrm{FBS}$. Transwell chambers were maintained at $37^{\circ} \mathrm{C}$. Twenty hours later, the cells in the basolateral chamber were stained with $0.1 \%$ crystal violet. Cells were observed, photographed, and counted under the inverted microscope. Transwell migration assays were performed in the absence of Matrigel, lasting for $16 \mathrm{~h}$.

\section{Microtube-forming assays}

Human brain microvascular endothelial cells (HBMVECs) were seeded into a Matrigel-coated 96-well plate with $2.5 \times 10^{4}$ cells for each well. Once adhered to the wall, HBMVECs were incubated with GC cell supernatant for 4-6 h.

\section{Fluorescence in situ hybridization (FISH)}

We used lncRNA subcellular localization data which are available at lncatlas.crg.eu. to predict subcellular localization of LINC00242. Then, FISH was performed to further examine the subcellular localization of LINC00242 using Ribo $^{\mathrm{TM}}$ IncRNA FISH Probe Mix (Red) (Ribo Biotech, Guangzhou, China) as per the manufacturers' instructions. In detail, cells were mounted onto slides and fixed in $4 \%$ formaldehyde. Slides were pretreated by protease $\mathrm{K}(2 \mu \mathrm{g} / \mathrm{mL})$, glycine and acetic anhydride, followed by prehybridization for $1 \mathrm{~h}$ at $42{ }^{\circ} \mathrm{C}$. Hybridisation was performed overnight at $42{ }^{\circ} \mathrm{C}$ using probes $(250 \mu \mathrm{L}, 300 \mathrm{ng} /$ $\mathrm{mL}$ ) against LINC00242. Finally, slides were stained with phosphate-buffered saline with Tween (PBST)-diluted 4',6-diamidino-2-phenyl indole (DAPI). Images were acquired with the fluorescence microscope (Olympus, Japan), with five random fields acquired from each slide.

\section{RNA immunoprecipitation (RIP)}

Cells were lysed in RIP Lysis Buffer at $4{ }^{\circ} \mathrm{C}$ for $30 \mathrm{~min}$. Cell extracts were incubated with protein A/G Sepharose beads conjugated with antibodies against Ago2 (P10502500, Otwo Biotech, Shenzhen, China) or normal mouse IgG. Immunoprecipitated RNA and total RNA from the whole cell lysates (input controls) were extracted for qRT-PCR analysis.

\section{Tumorigenicity assay in nude mice}

A total of $14 \mathrm{BALB} / \mathrm{c}$ nude mice (aged 4 weeks old and weighing 18-22 g) were purchased from Hunan SLAC Laboratory Animal Co., Ltd. (Changsha, China). Cell suspension $\left(5 \times 10^{7} / \mathrm{ml}\right)$ made from cells stably expressing shRNA targeting LINC00242 or scramble shRNA was subcutaneously injected into the nude mice to establish models of subcutaneous transplantation of GC in nude mice. Tumor growth was recorded every 3 days. Fifteen 
days later, mice bearing subcutaneous tumors were euthanized by cervical dislocation.

\section{Immunohistochemistry}

Paraffin-embedded sections $(5 \mu \mathrm{m})$ were dewaxed and hydrated and immunostained with the primary antibody, anti-VEGF (1:1000, ab53465, Abcam) or anti-CD31 (1:1000, ab134168, Abcam) at $4{ }^{\circ} \mathrm{C}$ overnight. Next, the sections were incubated with goat anti-rabbit Immunoglobulin G (IgG) (ab6721, 1:1000, Abcam) secondary antibody. Subsequently, the sections were developed by the diaminobenzidine (DAB) (ST033, Guangzhou Weijia Technology Co., Ltd., Guangzhou, China) and counterstained with hematoxylin (PT001, Shanghai Bogoo Biotechnology Co., Ltd., Shanghai, China) for $1 \mathrm{~min}$. Positive-stains were photographed under the microscope. Five high power fields were randomly selected in each slice (100 cells/field).

\section{Statistical analysis}

Results are presented as mean \pm standard deviation (s.d.) of three technical replicates. Student's $t$ test (paired or unpaired), one-way analysis of variance (ANOVA) followed by Tukey's test, and repeated measurements ANOVA with Bonferroni corrections were used for statistical comparisons as required. A value of $p<0.05$ indicated statistical significance using the SPSS 21.0 software (IBM Corp. Armonk, NY, USA).

\section{Results \\ LINC00242 was upregulated in GC tissues and cells}

We firstly obtained differential expression genes (DEGs) between GC tissues and normal tissues by analyzing raw data of GC-related expression datasets GSE79973, GSE19826 and GSE65801 that deposited in the Gene Expression Omnibus (GEO). Differential expression analysis was performed by using Bioconductor Project's (http://www.bioconductor.org) open-source software in $\mathrm{R}$ statistical programming language. Based on $\mathrm{p}$ value $(\mathrm{p}<0.05)$ and fold change (twofold as a cutoff), 1767, 707 and 2287 DEGs stood out from these three datasets, respectively. A heat map illustrating the top 50 DEGs is shown in Fig. 1a-c. Subsequent results (Fig. 1d) displayed 255 overlapping genes with significant difference in the three datasets, among which, there was one lncRNA, LINC00242, which was highly expressed in GC in the three datasets. Next, we collected clinical GC tissue and adjacent normal gastric tissue samples to detect the expression of LINC00242, and found that LINC00242 was abundantly expressed in GC tissues (Fig. 1e). The threshold of LINC00242 expression was obtained from the area under the receiver operating characteristic (ROC) curve. As shown in Fig. 1f, the area under the

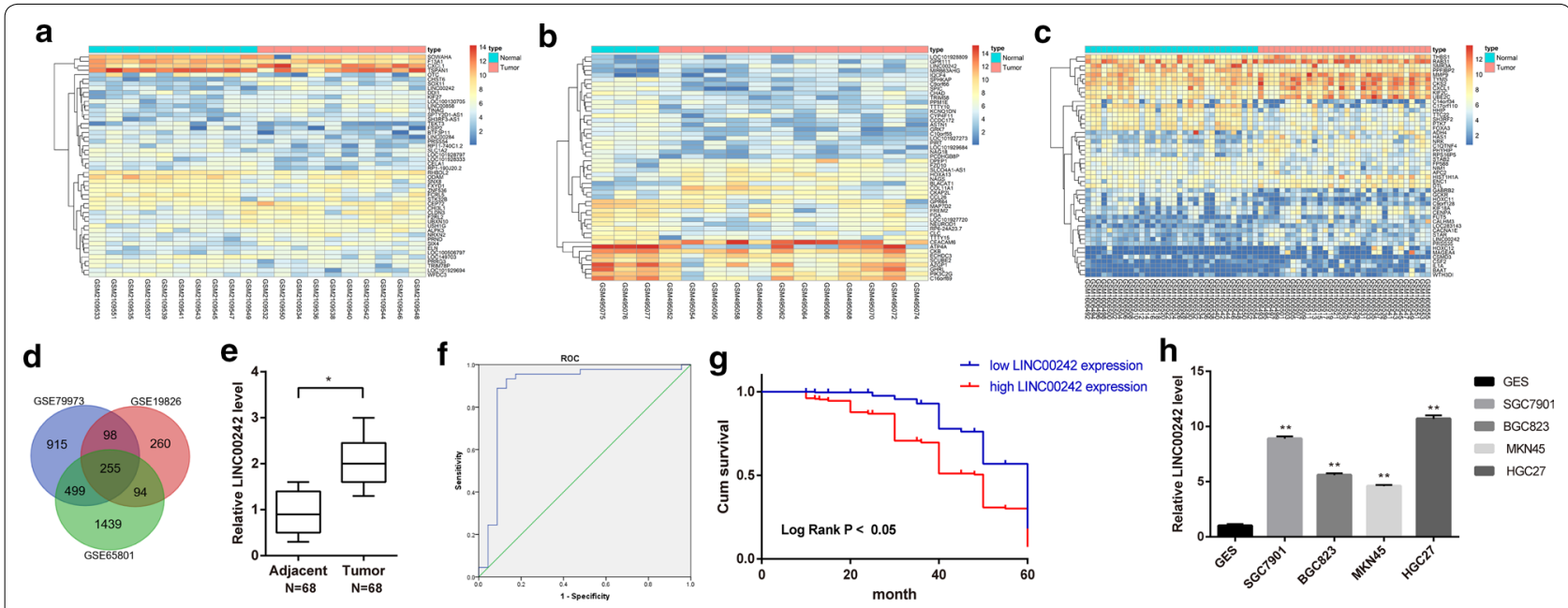

Fig. 1 Expression pattern of LINC00242 in GC tissues and cells. a-c, Heat maps depicting the DEGs obtained from the GC-related datasets GSE79973, GSE19826 and GSE65801. The abscissa represents the sample number, and the ordinate represents the differentially expressed gene; the left dendrogram represents gene expression cluster; each rectangle corresponds to a sample expression value; the histogram at the upper right refers to color gradation. $\mathbf{d}$ Venn analysis of DEGs in GC microarray, three circles in the figure respectively represent the DEGs from the three datasets, and the middle part represents the overlaps. e The expression of LINC00242 in 68 pairs of GC and adjacent normal gastric tissues was determined by RT-qPCR. $\mathbf{f}$ The area under the ROC curve according to LINC00242 expression. The threshold of LINC00242 expression was 0.803 . $\mathbf{g}$ The Kaplan-Meier method was used to plot GC patient survival according to LINC00242 expression. f The expression of LINC00242 in GES, SGC7901, BGC823, MKN45 and HGC27 cell lines was determined by RT-qPCR. Results are presented as mean \pm S.D. of three technical replicates. * (compared with adjacent normal gastric tissues) indicates $p<0.05$ by paired $t$-test. ${ }^{* *}$ (compared with GES cells) indicates $p<0.01$ by one-way ANOVA followed by Tukey's test 


\begin{tabular}{|c|c|c|c|c|c|}
\hline \multirow[t]{2}{*}{ Clinical characteristics } & \multirow[t]{2}{*}{ Case } & \multicolumn{2}{|c|}{$\begin{array}{l}\text { LINC00242 } \\
\text { expression }\end{array}$} & \multirow[t]{2}{*}{$x^{2}$} & \multirow[t]{2}{*}{$p$ value } \\
\hline & & high (n) & low (n) & & \\
\hline Gender & & & & 0.515 & 0.473 \\
\hline Male & 39 & 21 & 18 & & \\
\hline Female & 29 & 19 & 10 & & \\
\hline Age & & & & 0.020 & 0.451 \\
\hline$\leq 60$ & 48 & 29 & 19 & & \\
\hline$>60$ & 20 & 11 & 9 & & \\
\hline Lymph node metastasis & & & & 6.773 & $0.009^{*}$ \\
\hline No & 22 & 8 & 14 & & \\
\hline Yes & 46 & 32 & 14 & & \\
\hline TNM stage & & & & 10.105 & $0.018^{*}$ \\
\hline । & 13 & 4 & 9 & & \\
\hline$\|$ & 11 & 4 & 7 & & \\
\hline$\|$ & 20 & 15 & 5 & & \\
\hline IV & 24 & 17 & 7 & & \\
\hline Tumor differentiation & & & & 4.404 & $0.036^{*}$ \\
\hline High and medium & 23 & 9 & 14 & & \\
\hline Low and none & 45 & 31 & 14 & & \\
\hline Tumor size & & & & 1.060 & 0.303 \\
\hline$\leq 4 \mathrm{~cm}$ & 35 & 18 & 17 & & \\
\hline$>4 \mathrm{~cm}$ & 33 & 22 & 11 & & \\
\hline
\end{tabular}

Data were analyzed using Chi square test $\left(^{*} p<0.05\right)$
ROC curve is 0.894 , with cut-off value of 0.803 . Gastric cancer patients with LINC00242 expression of more than 0.803 were categorized into high-expressed group and those with LINC00242 expression of less than 0.803 were categorized into low-expressed group. Moreover, the results depicted in Table 2 showed that the expression of LINC00242 in GC was associated with the degree of tumor differentiation, lymph node metastasis and TNM stage $(p<0.05)$, but not with the gender, age and tumor size $(p>0.05)$. In addition, the overall 5 -year survival rate of GC patients with high LINC00242 expression was lower than that of GC patients with low LINC00242 expression (Fig. 1g). These results suggested that the high expression of LINC00242 in cancer tissues can predict the adverse prognosis of patients with GC. Subsequently, RT-qPCR analysis revealed that LINC00242 expression was upregulated in GC cell lines of SGC7901, BGC823, MKN45, HGC27 compared with normal gastric epithelial cell line GES $(p<0.05)$ (Fig. 1h), with the HGC27 cell lines showing the highest LINC00242 expression. Therefore, HGC27 cell lines were selected for the following experiments.

\section{LINC00242 knockdown represses GC cell migration, invasion, and in vitro angiogenesis}

In this part, we set out to study the effect of LINC00242 on the biological functions of GC cells. Firstly, we knock downed LINC00242 in HGC27 cells. To eliminate the influence of off-target, we set up three anti-LINC00242

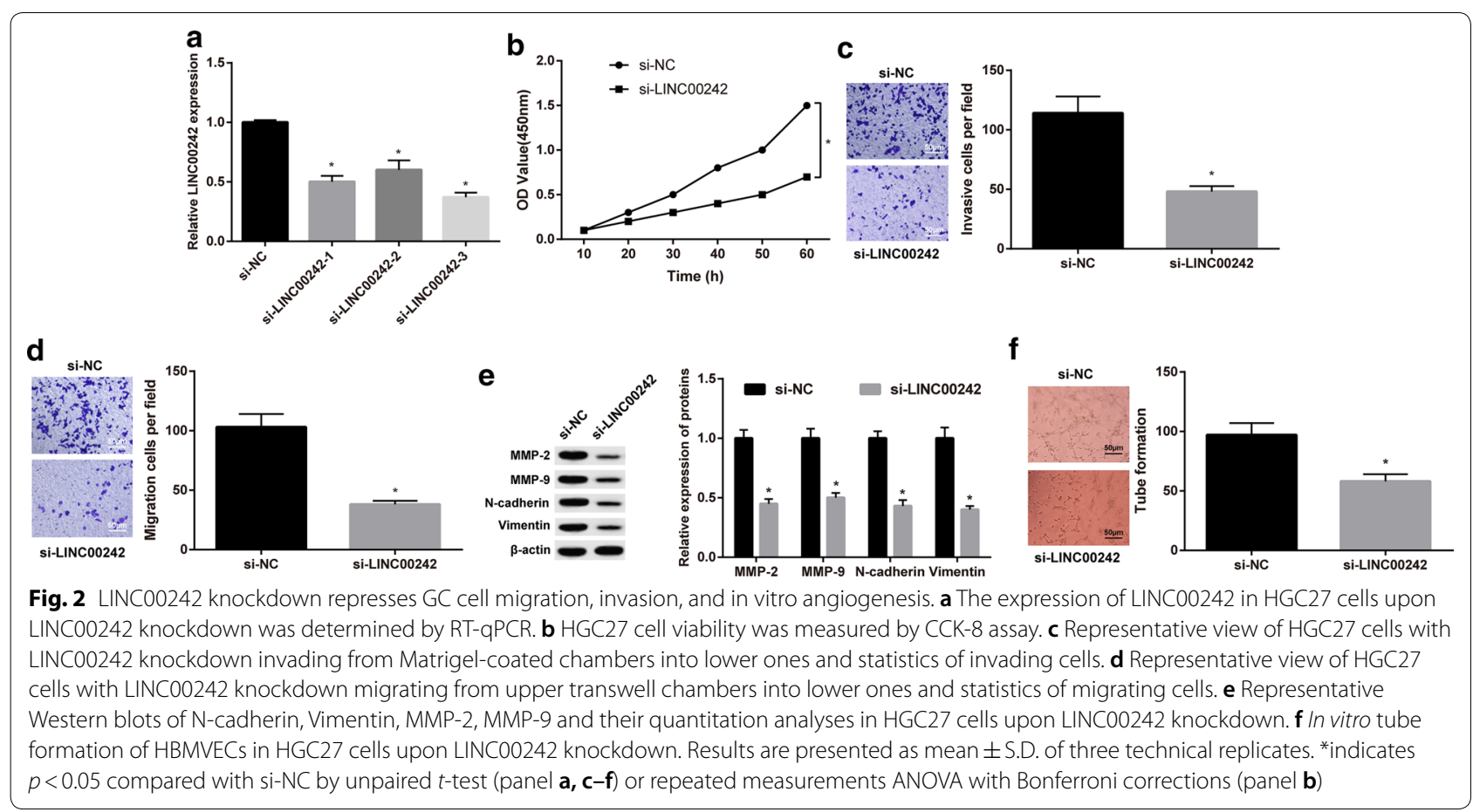


constructs, si-LINC00242-1, si-LINC00242-2 and si-LINC00242-3. As shown in Fig. 2a, all three antiLINC00242 constructs knock downed LINC00242 in HGC27 cells, whereas the si-LINC00242-3 knocked downed LINC00242 mostly. CCK-8 assay and Transwell chamber assays results revealed declined HGC27 cell viability, invasion, and migration upon LINC00242 knockdown $(p<0.05)$ (Fig. 2b-d). Western blot analysis showed decreased protein expression of N-cadherin, Vimentin, MMP-2 and MMP-9 in HGC27 cells upon LINC00242 knockdown $(p<0.05)$ (Fig. 2e). In addition, LINC00242 knockdown resulted in decreased tube formation of HBMVECs in HGC27 cell coculture (Fig. 2f). These data suggested that LINC00242 knockdown could inhibit GC cell migration, invasion, and in vitro angiogenesis.

\section{LINC00242 positively regulates FOXC1 expression by competitively binding to miR-141}

Next, we predicted the localization of LINC00242 and found that LINC00242 was expressed in both cytoplasm and nucleus, but mainly in cytoplasm (Fig. 3a). In order to further understand the mechanism of LINC00242 in GC, we retrieved GC-related GSE26595 dataset from the GEO database and found 41 significantly differentially expressed miRNAs (Fig. 3b). At the same time, 32 potential downstream miRNAs regulated by LINC00242 were obtained following prediction by the RAID database. Figure 3c revealed that there was one overlap miR-141 in the poorly expressed miRNAs from the GSE26595 dataset and the RAID database. We then analyzed another GSE13861 dataset retrieved from the GEO database and obtained 202 upregulated genes in GC (Fig. 3d). Concurrently, the downstream target genes of miR-141 were predicted by the mirDIP, TargetScan and DIANA databases. Then the first 200 genes predicted by the mirDIP (the score greater than 0.8), TargetScan and DIANA databases were selected and intersected with the downregulated genes from the GSE13861 dataset. The results shown in Fig. 3e revealed one overlapping FOXC1 gene. The above findings illustrated that LINC00242 is likely to regulate FOXC1 expression through miR-141, and ultimately affect the development of GC.

The FISH assay illustrated that LINC00242 was predominantly expressed in cytoplasm (Fig. 3f). The binding between miR-141 and LINC00242, miR-141 and FOXC1 detected by RIP assay is shown in Fig. 3g: LINC00242 and FOXC1 bound more to AGO2 antibody compared to IgG antibody, suggesting that miR-141 can specifically bind to LINC00242 and FOXC1. A biological prediction website (www.targetscan.org) revealed that miR-141 targeted and bound to FOXC1. The sequence of FOXC1 3'-UTR binding to miR-141 is illustrated in Fig. $3 \mathrm{~h}$. Dual-luciferase reporter gene assay results (Fig. 3i) showed that the luciferase activity of FOXC1-WT was reduced upon miR141 mimic transfection $(p<0.05)$ while that of FOXC1MUT showed no changes $(p>0.05)$. It was suggested that miR-141 could target FOXC1 and regulate its expression. In addition, RIP assay displayed that LINC00242 knockdown led to an increased expression of FOXC1 binding to AGO2 protein (Fig. 3j). RT-qPCR analysis showed a trend for increased miR-141 expression and decreased FOXC1 expression in HGC27 cells in response to si-LINC00242 transfection $(p<0.05) \quad$ (Fig. $3 \mathrm{k})$. Moreover, miR-141 expression was upregulated while FOXC1 expression was downregulated in HGC27 cells transfected with miR141 mimic $(p<0.05)$ (Fig. 3l), which further confirmed the results shown in Fig. 3i: miR-141 targets FOXC1 and negatively regulates its expression. Additionally, FOXC1 expression was found to be reduced in HGC27 cells with si-LINC00242+inhibitor NC $(p<0.05)$. However, siLINC00242 + miR-141 inhibitor presented with an opposite trend in FOXC1 expression $(p<0.05)$ (Fig. $3 \mathrm{~m})$. These data collected suggested LINC00242 interacted with miR-141 and positively regulated FOXC1, a target gene of miR-141.

\section{LINC00242 knockdown retards HGC27 cell viability, migration and invasion, and tube formation of HBMVECs by regulating miR-141 and FOXC1}

In order to further verify the effect of LINC00242 on the biological functions of $\mathrm{GC}$ by regulating $\mathrm{FOXC1}$ and miR-141, we firstly detected HGC27 cell viability by CCK-8 assay. The results revealed that HGC27 cell viability was accelerated upon transfection of si-LINC00242 coupled with miR-141 inhibitor or oe-FOXC1, suggesting miR-141 inhibition or FOXC1 overexpression negated the effect of LINC00242 knockdown on HGC27 cell viability $(p<0.05)$ (Fig. 4a). Transwell chamber assays revealed inhibited HGC27 cell invasion and migration upon transfection of si-LINC00242 coupled with miR141 inhibitor or oe-FOXC1, suggesting miR-141 inhibition or FOXC1 overexpression counteracted the effect of LINC00242 knockdown on HGC27 cell invasion and migration (Fig. 4b, c). Additionally, Western blot analysis demonstrated declined protein expression of MMP2, MMP-9, N-cadherin, and Vimentin in HGC27 cells following transfection of si-LINC00242 coupled with miR-141 inhibitor or oe-FOXC1, suggesting miR-141 inhibition or FOXC1 overexpression abrogated the effect of LINC00242 knockdown on these proteins $(p<0.05)$ (Fig. 4d). The subsequent results from tube-forming test showed that transfection of si-LINC00242 + miR-141 inhibitor and si-LINC00242+oe-FOXC1 resulted in enhanced tube formation of HBMVECs in HGC27 cell coculture (Fig. 4e). The aforementioned data revealed that LINC00242 knockdown could inhibit the viability, 


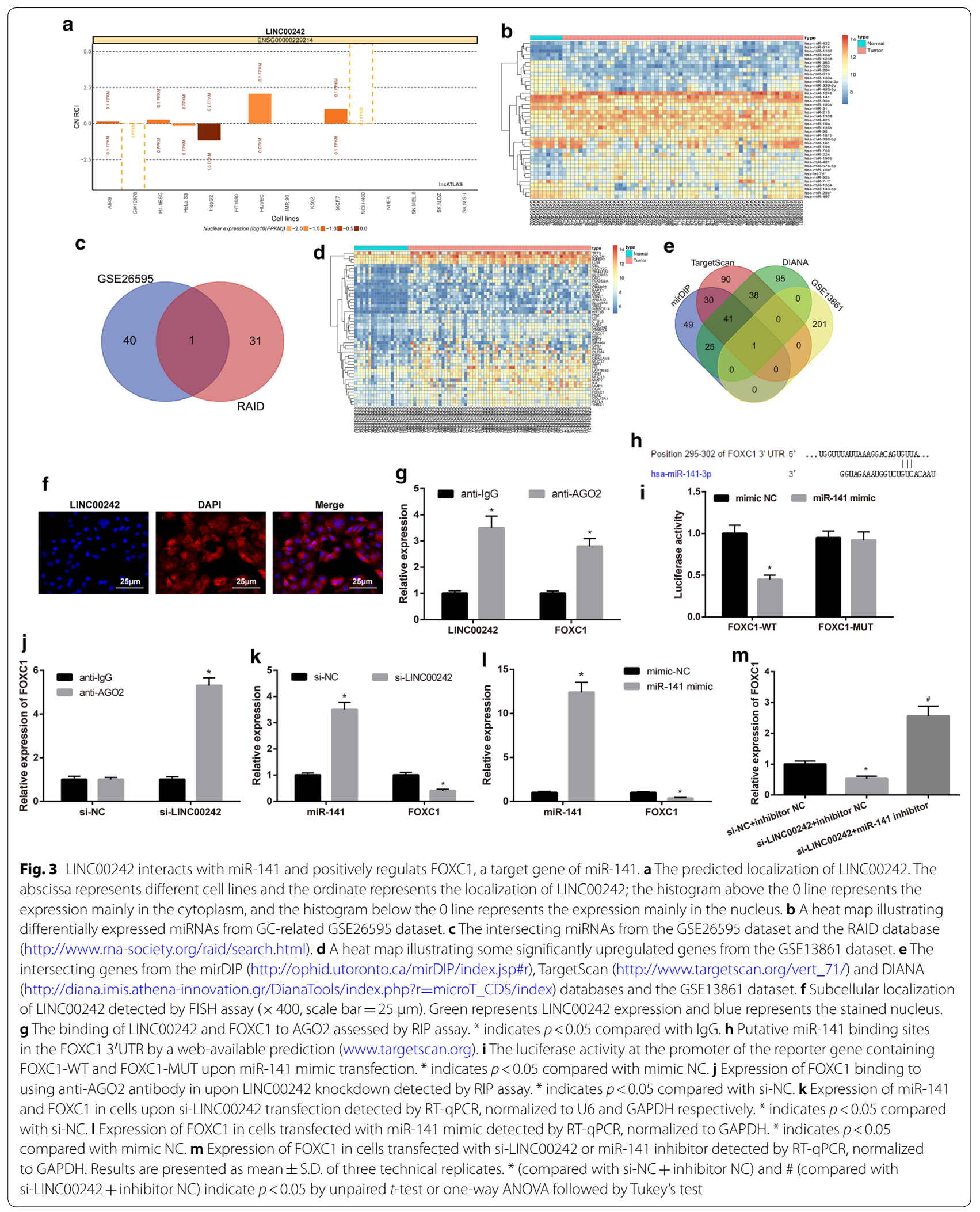




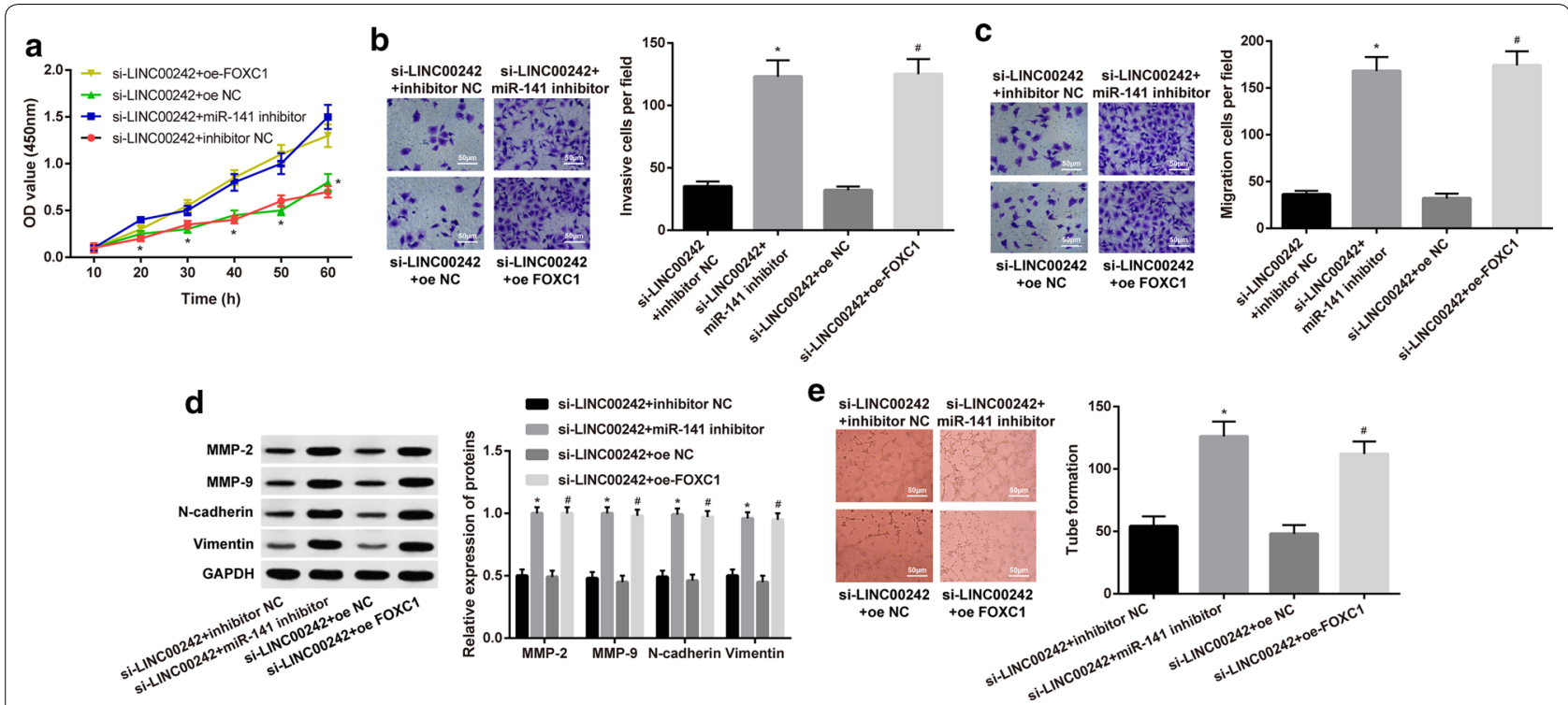

Fig. 4 LINC00242 knockdown retards HGC27 cell viability, migration and invasion, and tube formation of HBMVECs by regulating miR-141 and FOXC1. HGC27 cells were treated with si-LINC00242 alone or with miR-141 inhibitor or oe-FOXC1. a HGC27 cell viability was measured by CCK-8 assay. b Representative view of HGC27 cells invading from Matrigel-coated chambers into lower ones and statistics of invading cells. c Representative view of HGC27 cells migrating from upper transwell chambers into lower ones and statistics of migrating cells. $\mathbf{d}$ Representative Western blots of MMP-2, MMP-9, N-cadherin, Vimentin and their quantitation analyses in HGC27 cells. e In vitro tube formation of HBMVECs in HGC27 cells. Results are presented as mean \pm S.D. of three technical replicates. * (compared with si-LINC00242+inhibitor NC) and \# (compared with si-LINC00242+oe NC) indicate $p<0.05$ by unpaired $t$-test (panel $\mathbf{b}$-e) or repeated measurements ANOVA with Bonferroni corrections (panel $\mathbf{a}$ )

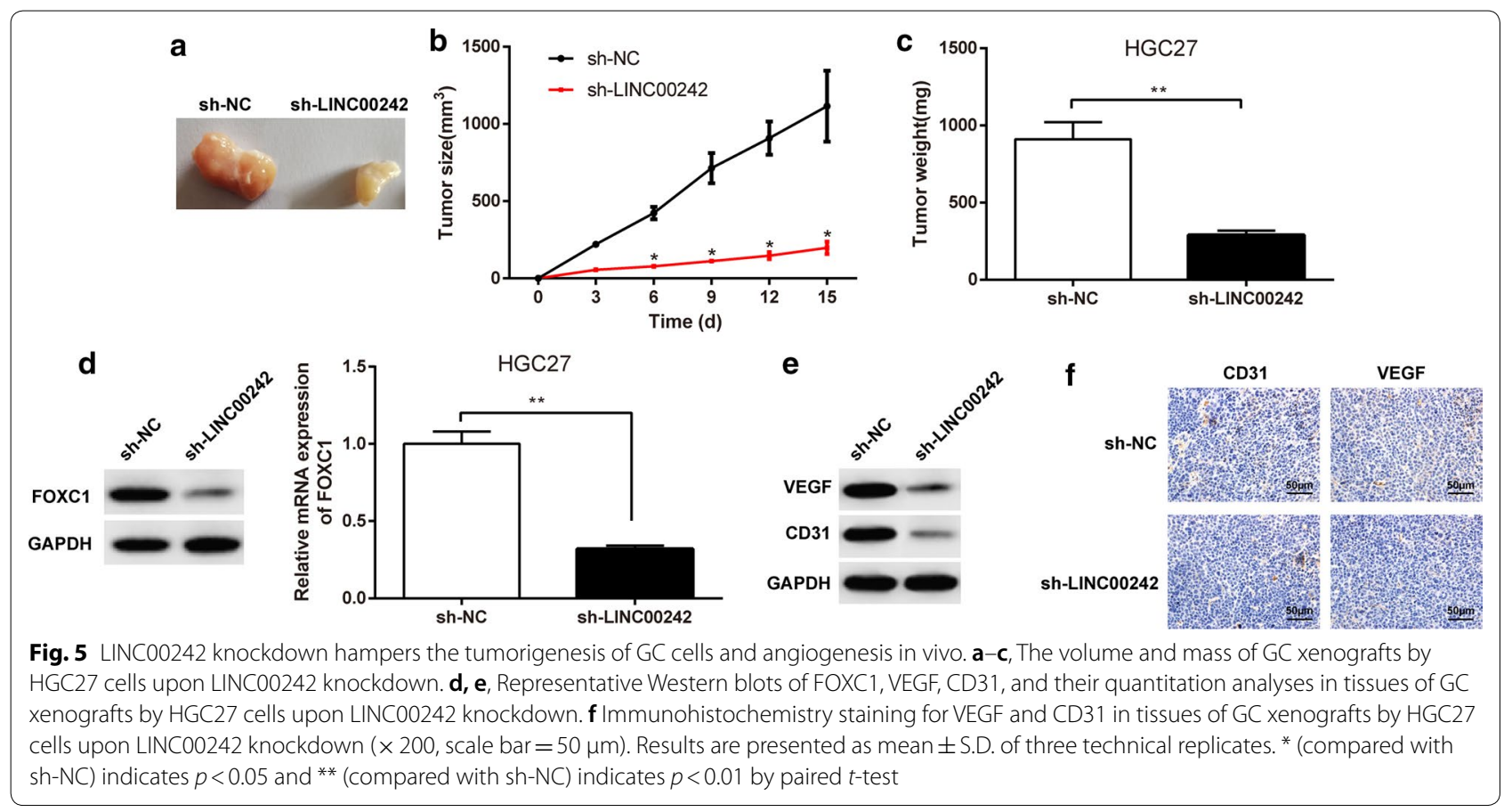


invasion, migration and tube formation of GC cells through promotion of miR-141 and inhibition of FOXC1.

\section{LINC00242 knockdown hampers the tumorigenesis of GC cells and angiogenesis in vivo}

Since we demonstrated that LINC00242 knockdown could repress GC cell growth, migration, invasion, and angiogenesis in vitro, we continued to verify its tumorsuppressive role in GC in vivo. As shown in Fig. 5a, the size of GC xenografts was decreased upon LINC00242 knockdown. The growth of GC xenografts in mice was monitored every 3 days after implantation duration of 15 days, and we observed attenuated tumor growth upon LINC00242 knockdown (Fig. 5b). The weight of GC xenografts was likewise declined upon LINC00242 knockdown (Fig. 5c). These data indicated that LINC00242 knockdown can inhibit the tumorigenesis of GC cells in vivo. Subsequent western blot analysis results revealed that the protein expression of FOXC1, VEGF and CD31 was declined in tissues of GC xenografts upon LINC00242 knockdown (Fig. 5d, e). We likewise observed declined immunohistochemistry staining for VEGF and CD31 proteins in tissues of GC xenografts following LINC00242 knockdown (Fig. 5f). These results demonstrated that LINC00242 could promote the tumorigenesis of GC cells and angiogenesis in vivo.

\section{Discussion}

Recently, the functions and mechanisms of lncRNAs have attracted increasing research attention either as tumor suppressors or oncogenes in prevalent cancers [15]. In the current investigation, the functional role of the LINC00242/miR-141/FOXC1 axis in the progression and metastasis of GC was explored. Collectively, our experimental data demonstrated that LINC00242 knockdown

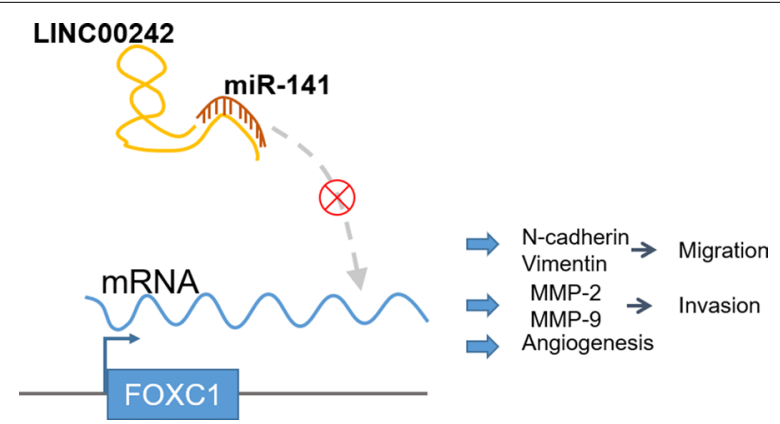

Fig. 6 Mechanism diagram illustrating the regulatory network and function of LINC00242 in GC. LINC00242 is upregulated in GC tissues. Overexpressed LINC00242 competitively binds to miR-141 and consequently promotes FOXC1 expression, thus increasing expression of N-cadherin, Vimentin, MMP-2 and MMP-9, ultimately accelerating the tumorigenesis and angiogenesis in GC exerted anti-tumor effects in GC by inhibiting malignant cell growth, migration, invasion and angiogenesis through miR-141 target-inhibition of FOXC1 (Fig. 6).

Fundamentally, our results identified the high expression of LINC00242 in GC tissues and cells in association with dismal oncological outcomes of patients. To our knowledge, the expression pattern or clinical significance of LINC00242 in any type of cancers at present still remain less studied, yet there are reports on other dysregulated lncRNAs in GC. A novel lncRNA, HOXC-AS3, has been reported to be upregulated in GC tissues corresponding to advanced tumor node metastasis (TNM) stage and lower overall survival [16]. Likewise, increased expression of LINC00673 and LINC00978 in GC has been confirmed to exert oncogenic effects in association with tumor size and TNM stage [17, 18]. Importantly, lncRNAs are of great significance to the occurrence, development and metastasis of cancer by regulating a spectrum of biological processes functioning as oncogenes or tumor suppressors [19]. Therefore, subsequent gain- or loss-of-function assays were performed to elucidate the action of deregulated LINC00242 in GC.

Further attempts were made to unravel the effects of aberrantly expressed LINC00242 in GC by delivering si-LINC00242 into HGC27 cells. It was observed that depletion of LINC00242 weakened the growth, migration, invasion and angiogenesis potential of HGC27 cells as evidenced by diminished levels of N-cadherin, Vimentin, MMP-2, MMP-9, VEGF and CD31. The tumorigenicity of HGC27 cells was also suppressed in the presence of depleted LINC00242. Expression of $\mathrm{N}$-cadherin, a biomarker of epithelial-mesenchymal transition, has been found to be elevated in GC samples in relation to lymph node metastasis, poor prognosis and advanced stage [20,21]. The function of Vimentin in GC has been evaluated as a prognostic marker that positive expression of Vimentin has the capacity to foreshadow metastasis and dismal prognosis [22, 23]. Low expression of MMP-2 and MMP-9 are highly suggestive of better survival condition of patients with GC $[24,25]$. Additionally, downregulation of MMP-2 and MMP-9 has been elaborated to involve in the antitumor activity of lysyl oxidase and secretory leukocyte protease inhibitor in GC $[26,27]$. Moreover, VEGF has been deciphered to play a part in the functional role of MMP-2 in GC [28]. Angiogenesis is of great therapeutic significance to GC [4]. As a major regulator of angiogenesis, VEGF is highly expressed in GC, facilitating the malignancies of tumor cells and growth and metastasis of tumors [29]. In addition to VEGF, CD31 expression is also elevated to be implicated in tumor angiogenesis of gastrointestinal stromal tumors that elevated CD31 expression is correlated with worse 
prognosis [30]. Similar regulatory mechanism with regard to lncRNA MNX1 antisense RNA 1 (MNX1AS1) has been unraveled that depleted lncRNA MNX1AS1 can reduce expression of Vimentin and MMP-9, supporting its oncologic role in GC [31].

Additionally, the possible underlying regulatory mechanism was studied and results revealed that LINC00242 worked in tandem with miR-141 and FOXC1 in GC. To be specific, LINC00242 could bind to miR-141 to mediate the expression of FOXC1, a target gene of miR-141. Interaction between IncRNAs and miRNAs has been recognized to harbor potential function in tumorigenesis, including GC [32-34]. Largely in agreement with our finding, LINC01234 has been indicated to serve as an oncogene in the progression of GC by modulating the miR-204-5p/core-binding factor $\beta$ axis [35]. Upregulation of LINC01296 has been demonstrated to exert growth-promoting effects in GC through interaction with miR-122 via MMP-9 [36]. Besides, miR-141 inhibition or overexpressed FOXC1 was shown to counterweigh the anti-tumor effects of LINC00242 knockdown in GC. By interacting with another lncRNA maternally expressed 3, miR-141 inhibition can offset its regulation on GC cell growth [37]. Also, overexpressed FOXC1 may be useful in predicting undesirable postoperative outcomes of patients with GC [38]. Notably, due to the limited time and funding, we were not allowed to investigate the exact expression patterns and functional roles of miR-141 and FOXC1 playing in GC, respectively, which requires further thorough research in the future for clinical rationale of the reported axis.

\section{Conclusion}

To conclude, these findings in the present investigation shed light on the carcinogenic action of LINC00242 in GC through its interaction with miR-141 and FOXC1, providing a novel therapeutic candidate to develop treatment modalities against GC. However, standard operation procedures are still expected for analytical validation and clinical utility of lncRNAs as promising biomarkers from different perspectives.

\footnotetext{
Abbreviations

LncRNAs: Long non-coding RNAs; miRNAs: microRNAs; GC: Gastric cancer; FOXC1: Forkhead box C1; RIP: RNA immunoprecipitation; HBMVECs: Human brain microvascular endothelial cells; FBS: Fetal bovine serum; RT-qPCR: Reverse transcription quantitative polymerase chain reaction; IgG: Immunoglobulin G; UTR: Untranslated region; FISH: Fluorescence in situ hybridization; ANOVA: One-way analysis of variance.
}

\section{Acknowledgements}

We would like show sincere appreciation to the reviewers for critical comments on this article.

\section{Authors' contributions}

$X Y$ and $X W$ are responsible for study design and manuscript editing, $L C$ and NG for data acquisition and data analysis, and XZ for literature research and manuscript preparation. All authors read and approved the final manuscript.

\section{Funding}

Not applicable.

\section{Availability of data and materials}

All data and materials are fully available without restriction. The data generated or analyzed during this study are included in this published article.

\section{Consent for publication}

All the authors agree to the publication clause.

\section{Competing interests}

None.

Received: 19 March 2020 Accepted: 19 June 2020

Published online: 24 June 2020

\section{References}

1. Bray F, Ferlay J, Soerjomataram I, Siegel RL, Torre LA, Jemal A. Global cancer statistics 2018: GLOBOCAN estimates of incidence and mortality worldwide for 36 cancers in 185 countries. CA Cancer J Clin. 2018:68:394-424.

2. Yuan DD, Zhu ZX, Zhang X, Liu J. Targeted therapy for gastric cancer: current status and future directions (Review). Oncol Rep. 2016;35:1245-54.

3. Rona KA, Schwameis K, Zehetner J, Samakar K, Green K, Samaan J, Sandhu K, Bildzukewicz N, Katkhouda N, Lipham JC. Gastric cancer in the young: an advanced disease with poor prognostic features. J Surg Oncol. 2017;115:371-5.

4. Blank S, Deck C, Dreikhausen L, Weichert W, Giese N, Falk C, Schmidt $\mathrm{T}$, Ott K. Angiogenic and growth factors in gastric cancer. J Surg Res. 2015;194:420-9.

5. Mashreghi M, Azarpara H, Bazaz MR, Jafari A, Masoudifar A, Mirzaei H, Jaafari MR. Angiogenesis biomarkers and their targeting ligands as potential targets for tumor angiogenesis. J Cell Physiol. 2018;233:2949-65.

6. Gu Y, Chen T, Li G, Yu X, Lu Y, Wang H, Teng L. LncRNAs: emerging biomarkers in gastric cancer. Future Oncol. 2015;11:2427-41.

7. Li Y, Wu Z, Yuan J, Sun L, Lin L, Huang N, Bin J, Liao Y, Liao W. Long non-coding RNA MALAT1 promotes gastric cancer tumorigenicity and metastasis by regulating vasculogenic mimicry and angiogenesis. Cancer Lett. 2017:395:31-44.

8. Wu WK, Lee CW, Cho CH, Fan D, Wu K, Yu J, Sung JJ. MicroRNA dysregulation in gastric cancer: a new player enters the game. Oncogene. 2010;29:5761-71

9. Du Y, Wang L, Wu H, Zhang Y, Wang K, Wu D. MicroRNA-141 inhibits migration of gastric cancer by targeting zinc finger E-box-binding homeobox 2. Mol Med Rep. 2015;12:3416-22.

10. Mao Y, Liu R, Zhou H, Yin S, Zhao Q, Ding X, Wang H. Transcriptome analysis of miRNA-IncRNA-mRNA interactions in the malignant transformation process of gastric cancer initiation. Cancer Gene Ther. 2017;24:267-75.

11. Zhou X, Ye F, Yin C, Zhuang Y, Yue G, Zhang G. The Interaction Between MiR-141 and IncRNA-H19 in regulating cell proliferation and migration in gastric cancer. Cell Physiol Biochem. 2015;36:1440-52.

12. XuY, Shao QS, Yao HB, Jin Y, Ma YY, Jia LH. Overexpression of FOXC1 correlates with poor prognosis in gastric cancer patients. Histopathology. 2014;64:963-70.

13. Aravalli RN, Greten TF. FoxC1: novel regulator of inflammationinduced metastasis in hepatocellular carcinoma. Gastroenterology. 2015;149:861-3.

14. PrasitsakT, Nandar M, Okuhara S, Ichinose S, Ota MS, Iseki S. Foxc1 is required for early stage telencephalic vascular development. Dev Dyn. 2015;244:703-11.

15. Prensner JR, Chinnaiyan AM. The emergence of IncRNAs in cancer biology. Cancer Discov. 2011;1:391-407. 
16. Zhang E, He X, Zhang C, Su J, Lu X, Si X, Chen J, Yin D, Han L, De W. A novel long noncoding RNA HOXC-AS3 mediates tumorigenesis of gastric cancer by binding to YBX1. Genome Biol. 2018;19:154.

17. Huang M, Hou J, Wang Y, Xie M, Wei C, Nie F, Wang Z, Sun M. Long noncoding RNA LINC00673 is activated by SP1 and exerts oncogenic properties by interacting with LSD1 and EZH2 in gastric cancer. Mol Ther. 2017;25:1014-26.

18. Fu M, Huang Z, Zang X, Pan L, Liang W, Chen J, Qian H, Xu W, Jiang P, Zhang X. Long noncoding RNA LINC00978 promotes cancer growth and acts as a diagnostic biomarker in gastric cancer. Cell Prolif. 2018;51:e12425.

19. $\mathrm{Li} \mathrm{CH}$, Chen Y. Insight into the role of long noncoding RNA in cancer development and progression. Int Rev Cell Mol Biol. 2016:326:33-65.

20. Okubo K, Uenosono Y, Arigami T, Yanagita S, Matsushita D, Kijima T, Amatatsu M, Uchikado Y, Kijima Y, Maemura K, Natsugoe S. Clinical significance of altering epithelial-mesenchymal transition in metastatic lymph nodes of gastric cancer. Gastric Cancer. 2017;20:802-10.

21. Kamikihara T, Ishigami S, Arigami T, Matsumoto M, Okumura H, Uchikado Y, Kita Y, Kurahara H, Kijima Y, Ueno S, Natsugoe S. Clinical implications of $\mathrm{N}$-cadherin expression in gastric cancer. Pathol Int. 2012;62:161-6.

22. Yin S, Chen FF, Yang GF. Vimentin immunohistochemical expression as a prognostic factor in gastric cancer: a meta-analysis. Pathol Res Pract. 2018;214:1376-80.

23. Otsuki S, Inokuchi M, Enjoji M, Ishikawa T, Takagi Y, Kato K, Yamada H, Kojima K, Sugihara K. Vimentin expression is associated with decreased survival in gastric cancer. Oncol Rep. 2011;25:1235-42.

24. Shen $\mathrm{W}, \mathrm{Xi} \mathrm{H}$, Wei $\mathrm{B}$, Chen $\mathrm{L}$. The prognostic role of matrix metalloproteinase 2 in gastric cancer: a systematic review with meta-analysis. J Cancer Res Clin Oncol. 2014;140:1003-9.

25. Yao Z, Yuan T, Wang H, Yao S, Zhao Y, Liu Y, Jin S, Chu J, Xu Y, Zhou W, Yang S, Liu Y. MMP-2 together with MMP-9 overexpression correlated with lymph node metastasis and poor prognosis in early gastric carcinoma. Tumour Biol. 2017:39:1010428317700411.

26. Zhao L, Niu H, Liu Y, Wang L, Zhang N, Zhang G, Liu R, Han M. LOX inhibition downregulates MMP-2 and MMP-9 in gastric cancer tissues and cells. J Cancer. 2019;10:6481-90.

27. Choi BD, Jeong SJ, Wang G, Park JJ, Lim DS, Kim BH, Cho YI, Kim CS, Jeong MJ. Secretory leukocyte protease inhibitor is associated with MMP-2 and MMP-9 to promote migration and invasion in SNU638 gastric cancer cells. Int J Mol Med. 2011;28:527-34.
28. Partyka R, Gonciarz M, Jalowiecki P, Kokocinska D, ByrczekT. VEGF and metalloproteinase 2 (MMP 2) expression in gastric cancer tissue. Med Sci Monit. 2012;18:BR130-4.

29. Chen S, Zhang X, Peng J, Zhai E, He Y, Wu H, Chen C, Ma J, Wang Z, Cai S. VEGF promotes gastric cancer development by upregulating CRMP4. Oncotarget. 2016;7:17074-86

30. Basilio-de-Oliveira RP, Pannain VL. Prognostic angiogenic markers (endoglin, VEGF, CD31) and tumor cell proliferation (Ki67) for gastrointestinal stromal tumors. World J Gastroenterol. 2015:21:6924-30.

31. Zhang W, Huang L, Lu X, Wang K, Ning X, Liu Z. Upregulated expression of MNX1-AS1 long noncoding RNA predicts poor prognosis in gastric cancer. Bosn J Basic Med Sci. 2019;19:164-71.

32. Karreth FA, Pandolfi PP. ceRNA cross-talk in cancer: when ce-bling rivalries go awry. Cancer Discov. 2013;3:1113-21.

33. Ma XY, Ma Y, Zhou H, Zhang HJ, Sun MJ. Identification of the IncRNAmiRNA-mRNA network associated with gastric cancer via integrated bioinformatics analysis. Oncol Lett. 2019;18:5769-84.

34. Pan H, Guo C, Pan J, Guo D, Song S, Zhou Y, Xu D. Construction of a competitive endogenous RNA network and identification of potential regulatory axis in gastric cancer. Front Oncol. 2019:9:912.

35. Chen X, Chen Z, Yu S, Nie F, Yan S, Ma P, Chen Q, Wei C, Fu H, Xu T, Ren S, Sun M, Wang Z. Long noncoding RNA LINC01234 functions as a competing endogenous RNA to regulate CBFB expression by sponging miR-204-5p in gastric cancer. Clin Cancer Res. 2018;24:2002-14.

36. Qin QH, Yin ZQ, Li Y, Wang BG, Zhang MF. Long intergenic noncoding RNA 01296 aggravates gastric cancer cells progress through miR-122/MMP-9. Biomed Pharmacother. 2018;97:450-7.

37. Zhou X, Ji G, Ke X, Gu H, Jin W, Zhang G. MiR-141 inhibits gastric cancer proliferation by interacting with long noncoding RNA MEG3 and downregulating E2F3 expression. Dig Dis Sci. 2015;60:3271-82.

38. Wu K, Fan Z, Xue Y, Yao Q, Zhang A. Rh(III)-catalyzed intermolecular C-H amination of 1-aryl-1H-pyrazol-5(4H)-ones with alkylamines. Org Lett. 2014;16:42-5.

\section{Publisher's Note}

Springer Nature remains neutral with regard to jurisdictional claims in published maps and institutional affiliations.

Ready to submit your research? Choose BMC and benefit from:

- fast, convenient online submission

- thorough peer review by experienced researchers in your field

- rapid publication on acceptance

- support for research data, including large and complex data types

- gold Open Access which fosters wider collaboration and increased citations

- maximum visibility for your research: over 100M website views per year

At BMC, research is always in progress.

Learn more biomedcentral.com/submissions 